\title{
Spatiotemporal dissipative solitons in two-dimensional photonic lattices
}

\author{
Dumitru Mihalache and Dumitru Mazilu \\ Horia Hulubei National Institute for Physics and Nuclear Engineering (IFIN-HH), \\ 407 Atomistilor, Magurele-Bucharest 077125, Romania \\ Falk Lederer \\ Institute of Solid State Theory and Theoretical Optics, Friedrich-Schiller Universität Jena, Max-Wien-Platz 1, D-07743 Jena, Germany
}

Yuri S. Kivshar

Nonlinear Physics Center, Research School of Physical Sciences and Engineering, Australian National University, Canberra, Australian Capital Territory 0200, Australia

(Received 3 June 2008; published 10 November 2008)

\begin{abstract}
We analyze spatiotemporal dissipative solitons in two-dimensional photonic lattices in the presence of gain and loss. In the framework of the continuous-discrete cubic-quintic Ginzburg-Landau model, we demonstrate the existence of novel classes of two-dimensional spatiotemporal dissipative lattice solitons, which also include surface solitons located in the corners or at the edges of the truncated two-dimensional photonic lattice. We find the domains of existence and stability of such spatiotemporal dissipative solitons in the relevant parameter space, for both on-site and intersite lattice solitons. We show that the on-site solitons are stable in the whole domain of their existence, whereas most of the intersite solitons are unstable. We describe the scenarios of the instability-induced dynamics of dissipative solitons in two-dimensional lattices.
\end{abstract}

DOI: 10.1103/PhysRevE.78.056602

PACS number(s): 05.45.Yv

\section{INTRODUCTION}

Nonlinear optical surface waves guided by interfaces separating different dielectric media were predicted and analyzed some time ago [1-8], but their direct observation has been hindered by experimental difficulties related to the high power thresholds required for their excitation. Recently, the interest in the study of nonlinear self-trapped optical surface waves has been renewed after the theoretical prediction $[9,10]$ and subsequent experimental demonstration [11] of nonlinearity-induced light localization near the edge of a truncated one-dimensional waveguide array with selffocusing nonlinearity, which can lead to the formation of the so-called discrete surface soliton. The generation of a surface soliton can be understood with the help of a simple physics [12] as a trapping of a discrete optical soliton [13] near the repulsive edge of the lattice when the beam power exceeds some threshold value. The surface solitons become possible solely due to the lattice discreteness effects, and they exist in neither continuous nor linear limits. Some of the specific features of such optical surface solitons in other relevant physical settings have been recently investigated both theoretically [14-18] and experimentally [19-23] (see also Ref. [24] for recent comprehensive overviews of experimental and theoretical developments in the area of discrete optical solitons).

Recently, we demonstrated theoretically that the presence of gain and loss, due to optical amplifiers and saturable absorbers, would affect the properties of discrete surface solitons [25]. Similar to other types of discrete dissipative solitons in both one- and two-dimensional lattices [26-29], the dissipative surface solitons exhibit novel features that, as a result of both discreteness and gain (loss) effects, have no counterpart in either the continuous limit or in other conservative discrete models for both cubic and quadratic nonlinear media [30-37].
Following our earlier studies $[25,38,39]$, in this paper we study the unique features of spatiotemporal GinzburgLandau (GL) solitons which can exist in both infinite and truncated two-dimensional photonic lattices in the presence of gain and loss. Theoretical studies of discrete surface solitons localized in the corners or at the edges of twodimensional photonic lattices [40-42] and recent observations of two-dimensional surface solitons in optically induced photonic lattices [43] and laser-written waveguide arrays in fused silica [44] demonstrated novel features of these two-dimensional nonlinear surface modes in comparison with their counterparts in one-dimensional waveguide arrays. In particular, in sharp contrast to one-dimensional discrete surface solitons, the mode threshold is lower at the surface than in a bulk making the mode excitation easier [41].

For our studies, we employ the GL equation, which is known to be a ubiquitous model in many physical problems [45], and in different forms it appears as the simplest model for describing dissipative solitons [46] and laser patterns in cavities [47]. In application to the photonic lattices, a periodic index modulation can be modeled by a discrete GL equation that describes the presence of gain and loss due to optical amplifiers and saturable absorbers [26]. Up to now, dissipative discrete solitons have been analyzed only in infinite one-dimensional arrays and lattices. In this paper, we study the properties of spatiotemporal GL solitons including surface solitons located in the corners or at the edges of the truncated two-dimensional photonic lattice in the presence of gain and loss. We find the domains of existence of such solitons in the relevant parameter space, for both on-site and intersite spatiotemporal dissipative solitons located in the corner, edge and the center of the lattice. We study the stability of spatiotemporal GL solitons and describe the com- 
plex instability-induced scenarios of their dynamics in twodimensional lattices.

\section{MODEL}

We consider light propagation in a square photonic lattice created by lossless weakly coupled arrays of identical evenly spaced two-dimensional homogeneous waveguides, which we model in the framework of the coupled-mode approach. In the discrete model the electric field is decomposed into modes of identical waveguides with the mode profile $\mathbf{e}\left(x, y, \omega_{0}\right)$, and the normalized (with respect to the coupling constant) propagation constant $k_{z}$ at the center frequency of the pulse $\omega_{0}$ as

$$
\mathbf{E}(x, y, z, t)=\sum_{n, m} E_{n, m}(t ; z) \mathbf{e}\left(x, y, \omega_{0}\right) \exp \left[i\left(k_{z} z-\omega_{0} t\right)\right]+\text { c.c. },
$$

where the propagation distance $z$ is normalized with respect to the coupling constant in both horizontal and vertical directions, and $t$ is the time normalized with respect to the ratio of group-velocity dispersion and coupling constant.

In the coupled-mode theory, only the amplitude $E_{n, m}$ of the electric field in the waveguide with the site $(n, m)$ is considered to evolve during propagation while the mode profile is assumed to remain constant. Therefore, the slowly varying normalized envelope $E_{n, m}$ obeys a set of coupled partial differential equations which describes, in the framework of the tight-binding approximation (or coupled-mode theory), the spatiotemporal dynamics of light in a twodimensional waveguide lattice in the presence of gain and loss,

$$
i \frac{\partial E_{n, m}}{\partial z}+\widetilde{D} \frac{\partial^{2} E_{n, m}}{\partial t^{2}}+\alpha\left(V_{n}+V_{m}\right) E_{n, m}+F\left(E_{n, m}\right)=0,
$$

where

$$
F(E)=i \delta E+\left(p+q|E|^{2}\right)|E|^{2} E .
$$

Here the lattice indices are $n, m=0,1, \ldots$, and $E_{-1, m}=E_{n,-1}$ $\equiv 0$ due to the lattice termination. We define the lattice couplings as $V_{n} E_{n, m}=E_{1, m}$ for $n=0, m \geqslant 0$ and $V_{n} E_{n, m}=E_{n+1, m}$ $+E_{n-1, m}$ for $n>0$, respectively, $V_{m} E_{n, m}=E_{n, 1}$ for $m=0, n$ $\geqslant 0$ and $V_{m} E_{n, m}=E_{n, m+1}+E_{n, m-1}$, for $m>0$.

In Eqs. (2) and (3) the real parameter $\delta>0$ stands for linear losses. Other coefficients of the discrete GinzburgLandau equation (2) are assumed, in general, to be complex, and they are defined as follows: $\widetilde{D}=D / 2-i \gamma, \alpha=\alpha_{r}+i \alpha_{i}, p$ $=\sigma-i \varepsilon$, and $q=\nu+i \mu$. We scale the real part of $\tilde{D}$ to $1 / 2$, i.e., we consider anomalous group-velocity dispersion (GVD) $(D=+1)$, and scale the self-focusing Kerr nonlinearity coefficient to $\sigma=+1$. The imaginary part $\gamma$ of the complex coefficient $\widetilde{D}$ accounts for the spectral filtering, i.e., dispersion of the linear loss. The real part $\alpha_{r}$ of the complex coefficient $\alpha$ is normalized to $1 / 2$; it accounts for tunneling between the adjacent waveguides, while the imaginary part $\alpha_{i}$ of the coefficient $\alpha$ stands for gain (losses) introduced by coupling. Here $\varepsilon>0$ is the nonlinear (cubic) gain, $\mu>0$ is the nonlin- ear (quintic) loss, while $\nu \leqslant 0$ accounts for the selfdefocusing quintic correction to the cubic (Kerr) nonlinearity (saturation of the optical nonlinearity).

Here we are restricting to the case of anomalous GVD $(D=+1)$ and self-focusing cubic nonlinearities $(\sigma=+1)$, because we are considering only the existence and stability of bright-type discrete spatiotemporal Ginzburg-Landau solitons. However, such bright-type discrete spatiotemporal solitons should also exist in the case of normal GVD $(D=-1)$ and for self-defocusing cubic nonlinearities $(\sigma=-1)$ [38]. In order to get stable bright-type solitons, a stable supporting zero background is necessary; therefore we have considered that the parameter $\delta$ accounting for linear losses is positive. By using the simple estimates given in Ref. [29], if the linear loss parameter $\delta$ is kept fixed, stable discrete spatiotemporal solitons exist in some domains in the parameter plane $(\varepsilon, \mu)$ if the following simple "rule of thumb" holds: $\varepsilon^{2} \geqslant 4 \mu \delta$ (see Fig. 8 below).

\section{SPATIOTEMPORAL SOLITONS}

We look for spatiotemporal localized solutions of the discrete GL equations (2) and (3) in the form

$$
E_{n, m}(t ; z)=E_{n, m}(t) \exp (i \beta z),
$$

where $\beta$ is the nonlinearity-induced shift of the waveguide propagation constant, and the envelope $E_{n, m}(t)$ describes the temporal evolution of the solitonlike pulse propagating in the $(n, m)$ waveguide lattice site. Although in a discrete model various combinations of the signs of dispersion and nonlinearity as well as the spatial topology may potentially lead to different types of spatiotemporal localized solutions, in this paper we restrict ourselves, for the sake of clarity and simplicity, to the case of anomalous GVD $(D=+1)$, selffocusing nonlinearity $(\sigma=+1)$, and in-phase (unstaggered) localized modes in two-dimensional dissipative lattices.

The localized solutions $E_{n, m}(t)$ of the partial differential equations (2) are found by adequate numerical methods assuming that the amplitude of the pulses in each waveguide, $\max \left|E_{n, m}\right|$, decays rapidly far from the corners and the edges of the truncated waveguide lattice, so that the corresponding solution describes a spatiotemporal mode localized in the lattice corner or at its edge. Also we find numerically the nonlinear discrete spatiotemporal GL solitons located far away from the corners and edges, so such modes can be therefore treated as the modes of an infinite two-dimensional photonic lattice.

For the numerical calculations we fix, for simplicity, some of the parameters of the GL model as follows: $\gamma=0, \alpha_{i}=0$, $\nu=-0.1, \delta=2$, and vary the nonlinear (cubic) gain $\varepsilon$ and the nonlinear(quintic) loss $\mu$. In order to find numerically the envelope $E_{n, m}(t)$ of the spatiotemporal dissipative soliton we start with an arbitrary input pulse (typically, a Gaussian one), and simulate Eqs. (2) and (3) forward in $z$, expecting that a stable dissipative soliton localized at the corner, edge or deep inside the photonic lattice will emerge after a certain propagation distance in the form of $E_{n, m}(t ; z)=E_{n, m}(t) \exp (i \beta z)$, where the propagation constant $\beta$ is the corresponding eigenvalue determined by the parameters of Eqs. (2) and (3). As in 

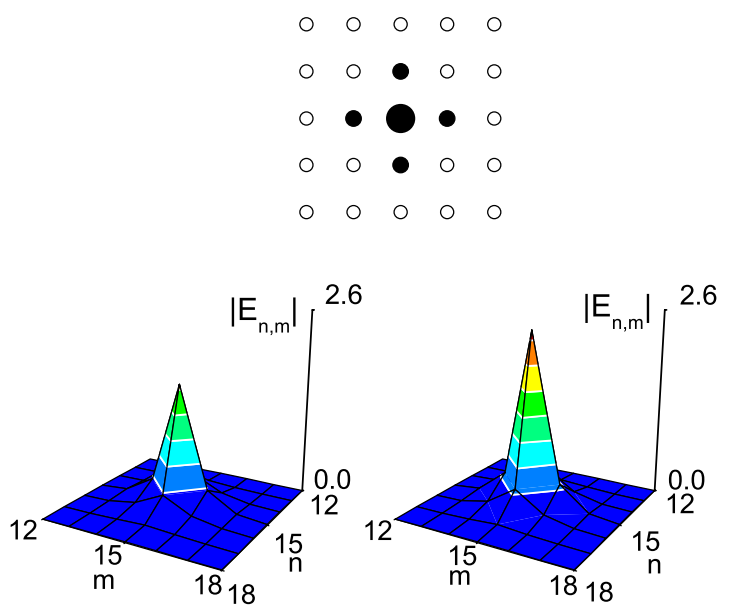

FIG. 1. (Color online) Spatial cross sections of stable on-site spatiotemporal solitons with low and high peak amplitudes localized in the center of the lattice. Parameters are $\mu=1$ and $\varepsilon=3.5$ (left), $\mu=0.2$ and $\varepsilon=1.5$ (right).

the case of spatiotemporal surface GL solitons in onedimensional photonic lattices [25], we employ a standard Crank-Nicolson scheme for numerical integration of the coupled complex GL equations; the temporal grid has a typical step length $\Delta t=0.1$, whereas the typical longitudinal step size is $\Delta z=0.01$. Depending on the value of the propagation constant, we use up to 161 discretization points in the continuous time interval $\left[0, t_{\max }\right]$, and up to $31 \times 31$ grid points for the discrete spatial coordinates. The nonlinear finitedifference equations are solved by using the Picard iteration method [48], and the resulting linear system is handled with the help of the Gauss-Seidel iterative procedure. To achieve good convergence, we typically need ten Picard and four Gauss-Seidel iterations. The propagation constant $\beta$ is determined as the $z$ derivative of the phase of $E_{n, m}(t ; z)$, and the solution is reckoned to achieve a stationary form if $\beta$ ceases to depend on $z$ and $t$, up to five significant digits.

Typical examples of spatial profiles and temporal crosssections of stable on-site (odd) spatiotemporal GL solitons localized in the center of the lattice (representing a spatiotemporal discrete soliton in an infinite two-dimensional lattice), together with the surface solitons located in the corners or at the edges of the truncated photonic lattice, are shown in Figs. 1-3 for two representative sets of the parameters $(\mu, \varepsilon)$. These nonlinear spatiotemporal continuousdiscrete dissipative localized states can be termed "dissipative discrete surface light bullets" [49,50]. We mention that similar shapes of the on-site solitons are obtained for other parameter sets, too. The overall rule is the following: when the nonlinear quintic loss $\mu$ is fixed the amplitude (and energy) of the soliton increases as the nonlinear cubic gain $\varepsilon$ grows, whereas when the cubic gain parameter is fixed the amplitude (and energy) of the soliton increases as the quintic loss parameter decreases.

The localized modes centered in the corners or at the edges of the two-dimensional square photonic lattice which are found here provide a generalization to dissipative dynamical systems of the spatiotemporal surface solitons recently predicted to exist in nondissipative (Hamiltonian)
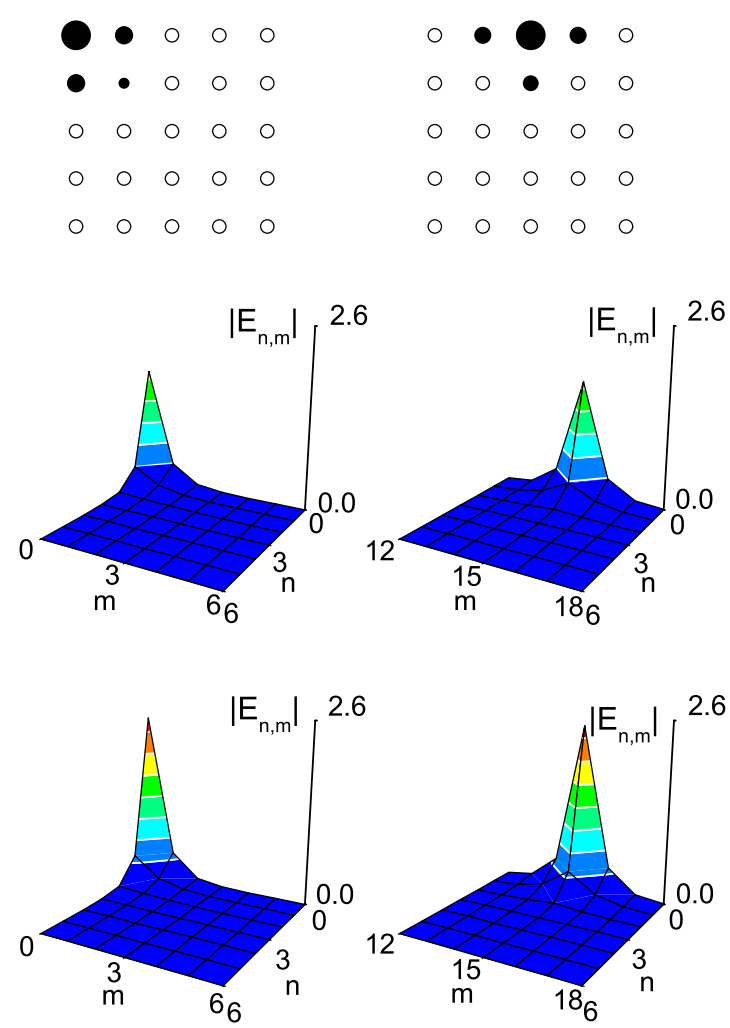

FIG. 2. (Color online) Examples of spatial cross sections of stable on-site spatiotemporal surface solitons with both relatively low and relatively high peak amplitudes localized in the lattice corner (left column) and at the lattice edge (right column). Parameters are $\mu=1$ and $\varepsilon=3.5$ (for low-amplitude solitons); $\mu=0.2$ and $\varepsilon$ $=1.5$ (for high-amplitude solitons).

semi-infinite discrete dynamical systems [38,39,51,52]. Importantly, the central mode located deep inside the center of the lattice, representing a continuous-discrete spatiotemporal GL soliton in an infinite two-dimensional waveguide lattice, has not been studied before, to the best of our knowledge.

In Fig. 4 we show typical examples of spatial cross sections of stable on-site corner [localized in the $(1,1)$ lattice site] and edge [localized in the $(1,15)$ lattice site] spatiotemporal surface GL solitons with both relatively low and relatively high peak amplitudes corresponding to two sets of parameters $(\mu, \varepsilon)$. Several examples of spatial cross sections of double-peak intersite spatiotemporal surface solitons located in the corners or at the edges of the truncated lattice, together with the central mode located deep inside the lattice, are shown in Fig. 5. The double-peak intersite solitons are unstable in large domains of the parameter space; however, we have found that in some restricted domains of the relevant parameters $(\mu, \varepsilon)$, the double-peak intersite bulk solitons (the central modes) are found to be stable (see below the detailed stability analysis). Only the high-peak-amplitude (and energy) intersite soliton located in the center of the lattice shown in Fig. 5(f) was found to be stable [see also Figs. 10(e) and 10(f)]. We mention that the temporal profiles corresponding to the spatial profiles shown in Figs. 4 and 5 are similar to those displayed in Fig. 3.

The dissipative optical solitons can be characterized by the total energy $\mathcal{E}$ defined as 


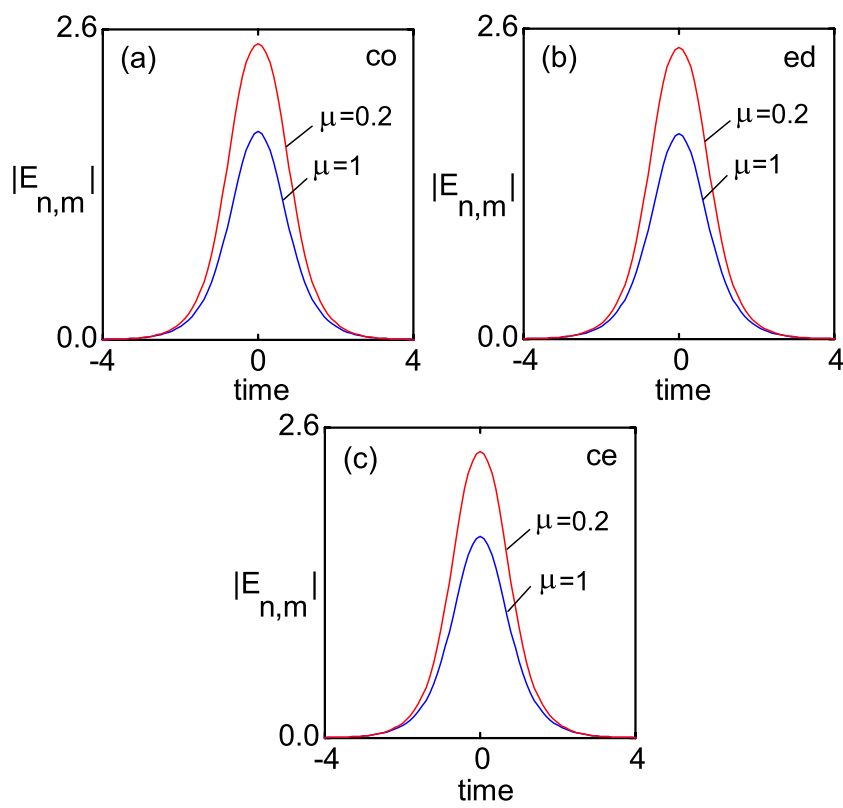

FIG. 3. (Color online) Temporal cross sections of stable on-site spatiotemporal surface solitons localized in the corner, at the edge, and in the center of the lattice, respectively. Shown are the temporal profiles of relatively low-amplitude and relatively high-amplitude solitons corresponding to the spatial profiles displayed in Figs. 1 and 2. Here $\varepsilon=3.5$ (for low-amplitude solitons) and $\varepsilon=1.5$ (for high-amplitude solitons)

$$
\mathcal{E}=\sum_{n, m} \int_{-\infty}^{+\infty}\left|E_{n, m}(t)\right|^{2} d t,
$$

which remains constant for stationary solitons. The dependencies $\mathcal{E}=\mathcal{E}(\varepsilon)$ and $\beta=\beta(\varepsilon)$ for the corner and edge on-site and double-peak intersite surface solitons are shown in Fig. 6. In Fig. 7 we show the normalized energy $\mathcal{E}$ and propagation constant $\beta$ vs cubic gain $\varepsilon$, for both relatively lowenergy (for $\mu=1$ ), and relatively high-energy (for $\mu=0.2$ ) on-site solitons located far away from the lattice edges. If we compare the corresponding energy curves of different surface modes, including the case of a spatiotemporal soliton located inside the lattice, we notice that in a large domain of variation of the nonlinear (cubic) gain $\varepsilon$, the energy of surface modes localized in the corners and at the edges of the lattice is lower than that of the bulk mode, similar to the case of surface light bullets in the corresponding conservative system [52].

\section{STABILITY ANALYSIS}

We find that the on-site spatiotemporal dissipative solitons located in the corner (co), at the edge (ed), and in the center (ce) of the lattice are stable in some intervals of the variation of the cubic gain $\varepsilon$ (see Fig. 8). In fact, we find that there exists a minimum value $\varepsilon_{\text {low }}$ of the nonlinear gain $\varepsilon$ for supporting a stable soliton. Indeed, for the values of $\varepsilon$ smaller than the minimum value $\varepsilon_{\text {low }}$ the input pulse decays whereas for the values of the cubic gain parameter inside the stability interval $\left[\varepsilon_{\text {low }}, \varepsilon_{\text {upp }}\right]$ the Gaussian input rapidly converges to-
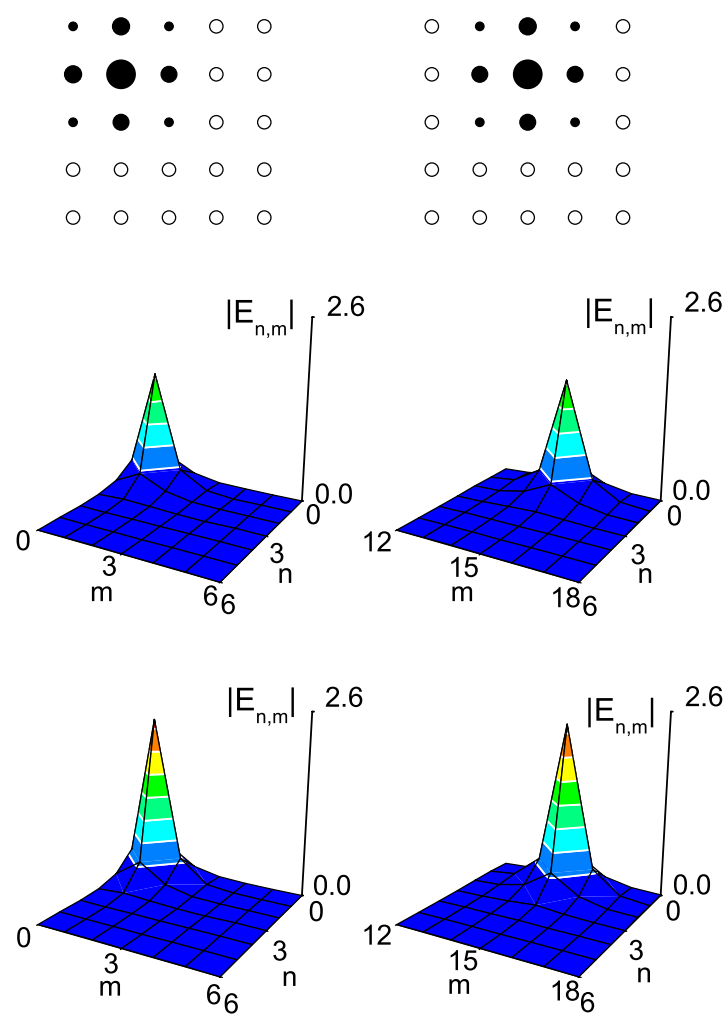

FIG. 4. (Color online) Examples of spatial cross sections of stable on-site corner [localized in the $(1,1)$ lattice site] and edge [localized in the $(1,15)$ lattice site] spatiotemporal surface solitons with both relatively low and relatively high peak amplitudes. Here $\mu=1$ and $\varepsilon=3.5$ (for low-amplitude solitons), and $\mu=0.2$ and $\varepsilon$ $=1.5$ (for high-amplitude solitons).

ward the corresponding on-site discrete surface soliton, and its propagation constant $\beta$ can be uniquely determined. However, for values of the nonlinear gain parameter $\varepsilon$ larger than a maximum value $\varepsilon_{\text {upp }}$, a dissipative soliton cannot be formed as well; in this case the soliton propagation constant $\beta$ cannot be determined numerically (the peak amplitude and the energy increase indefinitely in an oscillatory manner). The Gaussian input is converted into an expanding pattern composed of two fronts moving in opposite directions along the temporal axis, similar to the case of dissipative spatiotemporal surface solitons in one-dimensional waveguide arrays [25]. We mention that stability domains similar to those shown in Figs. 8(a) and 8(b) for the $(0,0)$ corner and $(0,15)$ edge solitons have been found for the $(1,1)$ corner and $(1,15)$ edge solitons as well.

The stability of the on-site (odd) and intersite (even) spatiotemporal surface GL solitons has been investigated by performing a linear stability analysis (via the calculation of the perturbation eigenvalues) and by double-checking these results in direct propagation simulations. To this end, we have considered a perturbed solution of the nonlinear dynamical system (2) of the form

$$
E_{n, m}(t ; z)=\left[E_{n, m}(t)+f_{n, m}(t) \exp (\lambda z)\right] \exp (i \beta z),
$$

where $\lambda$ (which may be, in general, a complex number) is the growth rate of the small perturbation represented by the eigenmode $f_{n, m}$. 

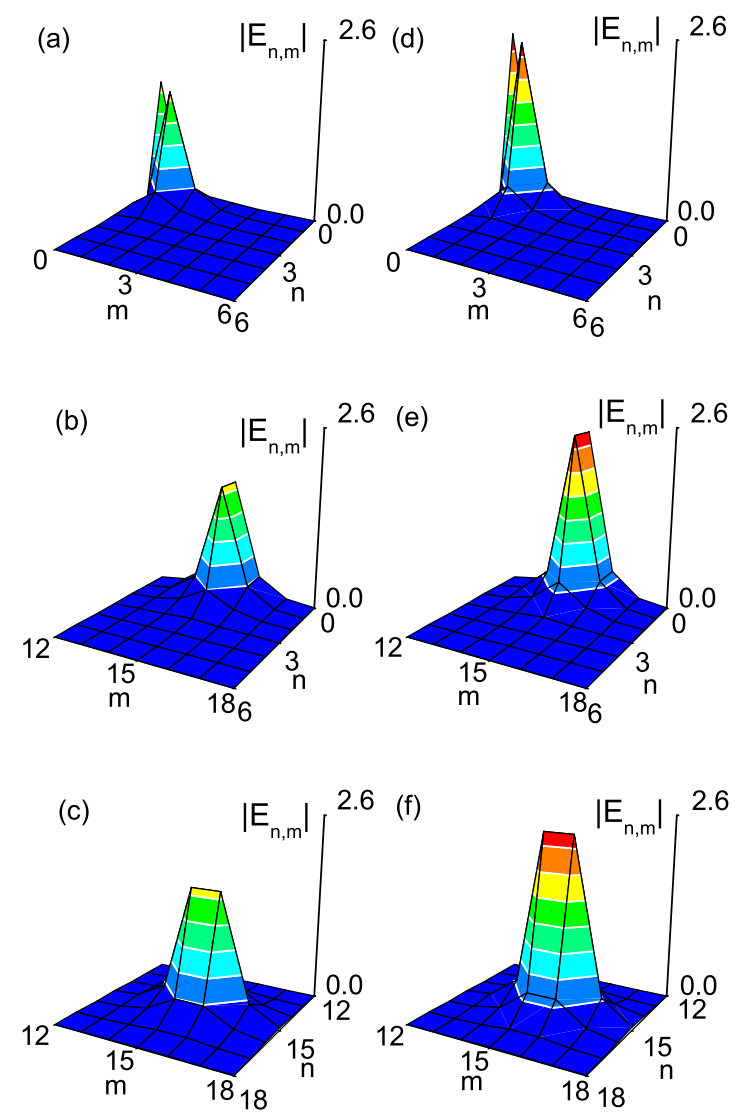

FIG. 5. (Color online) Examples of spatial cross sections of double-peak intersite spatiotemporal surface solitons with both low and high peak amplitudes localized in the lattice corner (a) and (d), at the edge (b) and (e), and in the center of the lattice (c) and (f). Here $\mu=1$ and $\varepsilon=3.5$ in (a)-(c), and $\mu=0.2$ and $\varepsilon=1.5$ in (d)-(f).

The substitution of the above expression into the coupled system of GL equations [2] leads to linearized equations for the perturbation eigenmodes $f_{n, m}$ and perturbations eigenvalues $\lambda$, which were solved by standard numerical techniques. We have found that for the on-site solitons $\max \operatorname{Re}(\lambda)=0$, implying their stability in the whole domain of their existence, whereas double-peak corner and edge intersite solitons were found to be unstable in the whole domain of their existence, because $\max \operatorname{Re}(\lambda)>0$ (see Fig. 9 for some typical examples for $\mu=1$ ). The stability of multiple-peaked intersite GL solitons located deep in the center of the twodimensional lattice deserves a separate study; here we present only a few typical examples. Though the on-site center solitons were found to be stable in the whole domain of their existence [see Figs. 10(a) and 10(b)], the two-peaked intersite center solitons were found to be either stable [see Figs. 10(e) and 10(f)] or unstable [see Figs. 10(c) and 10(d)], depending on the values of the two relevant parameters of the GL model, namely, $\mu$ and $\varepsilon$. We expect to get also domains of stability for four-peaked intersite spatiotemporal GL solitons located deep inside the lattice, similar to the case of spatial discrete GL solitons in two-dimensional lattices [29].
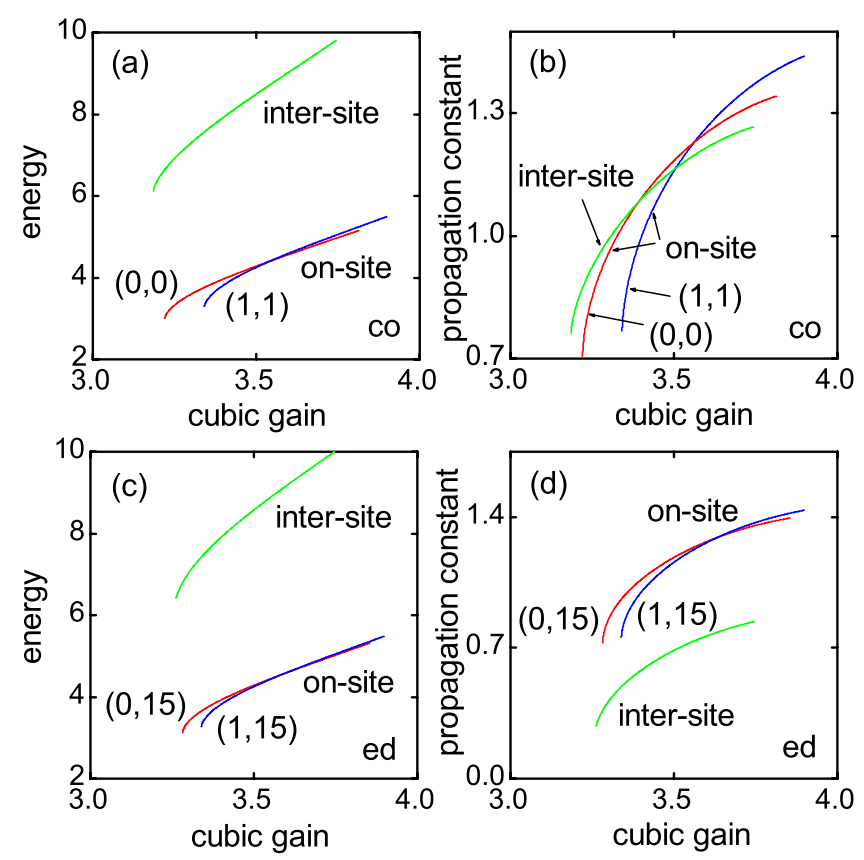

FIG. 6. (Color online) (a),(c) Normalized energy $\mathcal{E}$ vs cubic gain $\varepsilon$, and (b),(d) propagation constant $\beta$ versus cubic gain $\varepsilon$, for (a),(b) corner and (c),(d) edge on-site and double-peak intersite surface solitons. Here $\mu=1$.

Notice that, in addition to the fundamental on-site $(0,0)$ corner soliton, other on-site corner solitons centered in the $(1,1)$ and $(2,2)$ lattice sites are found to be stable in their whole existence domain [see Fig. 6(a), where the energycubic gain curves are shown only for the cases of $(0,0)$ and $(1,1)$ corner solitons because the corresponding curve for $(2$,
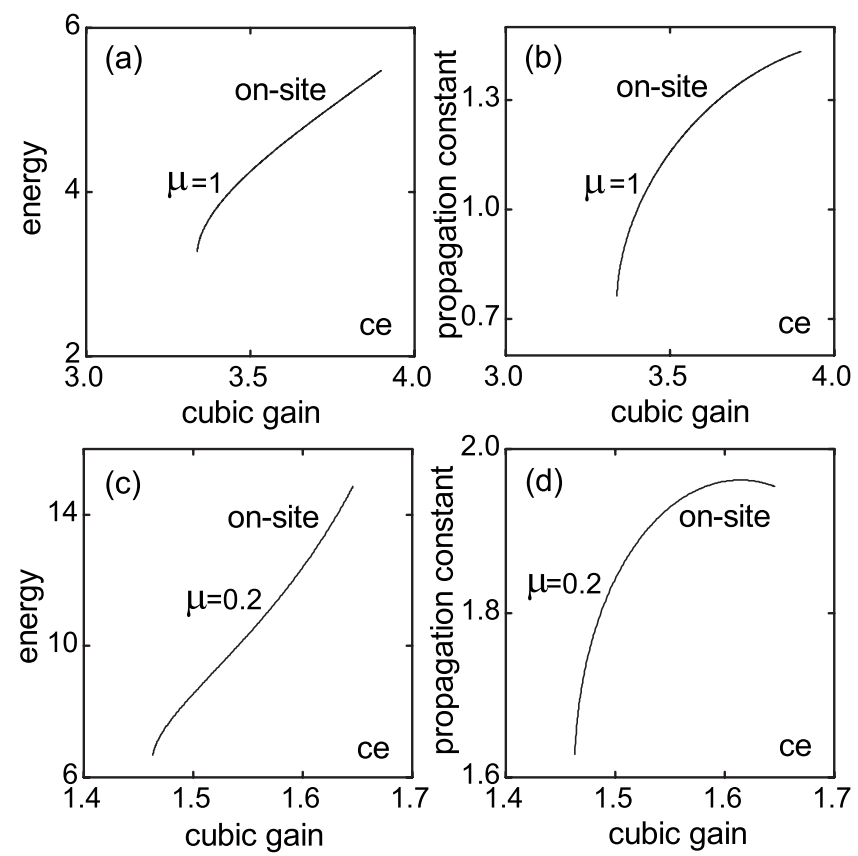

FIG. 7. (a),(c) Normalized energy $\mathcal{E}$ vs cubic gain $\varepsilon$, and (b),(d) propagation constant $\beta$ versus cubic gain $\varepsilon$, for (a),(b) relatively low-energy (for $\mu=1$ ), and relatively high-energy (for $\mu=0.2$ ) onsite solitons localized deep in the center of the lattice. 

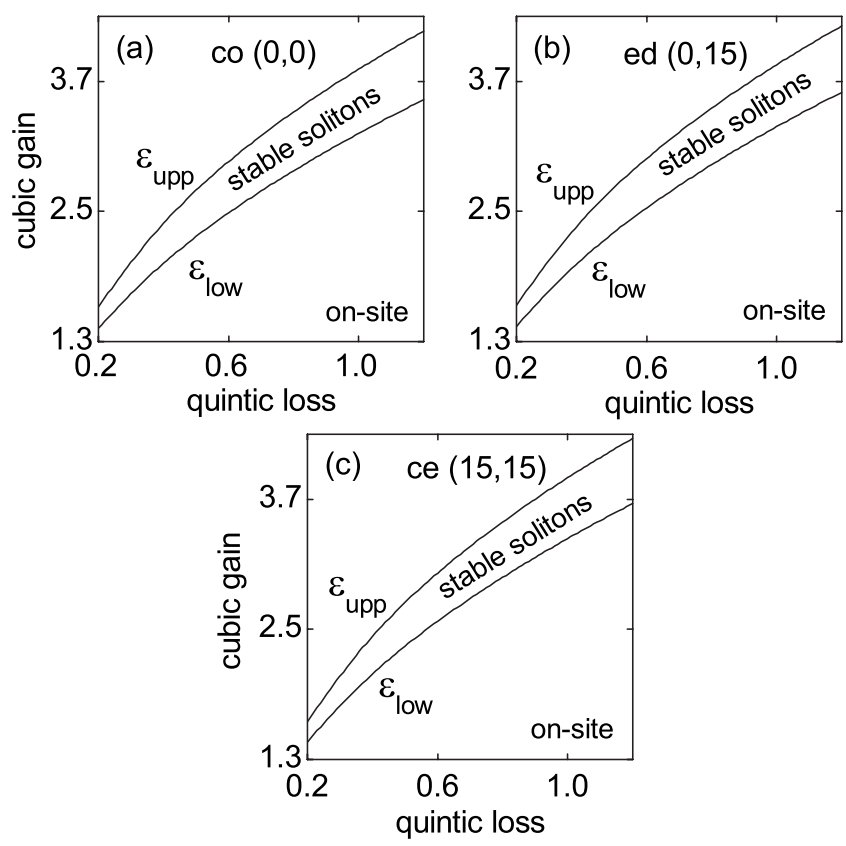

FIG. 8. Domains of existence and stability in the plane $(\mu, \varepsilon)$ for on-site dissipative discrete light bullets located in the corner (co), at the edge (ed), and in the center (ce), respectively.

2) solitons nearly overlaps them]. Moreover, we have found that, in addition to the fundamental on-site $(0,15)$ edge soliton, other on-site edge solitons centered in the $(1,15)$ and $(2$, 15) lattice sites are found to be stable in their whole existence domain [see Fig. 6(c), where the energy-cubic gain curve for $(2,15)$ solitons is not shown because it nearly overlaps the corresponding $(0,15)$ and $(1,15)$ curves]. Moreover, we have found that the out-of-phase (staggered) discrete spatiotemporal Ginzburg-Landau solitons are unstable.

\section{INSTABILITY-INDUCED DYNAMICS}

To cross-check the results of our linear stability analysis, we employ direct numerical simulations to study the propagation dynamics of both stable and unstable solitons. We find that the on-site dissipative solitons survive in the presence of a relatively large (up to $10 \%$ ) white input noise added to the
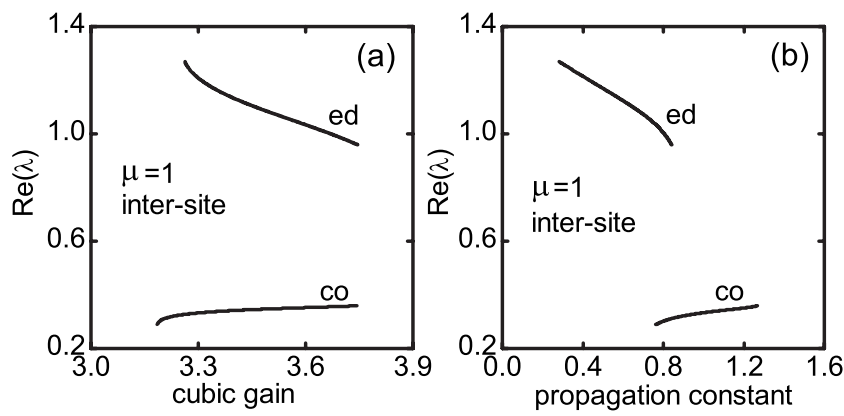

FIG. 9. Largest instability growth rate versus cubic gain (a) and versus propagation constant (b) for double-peak intersite corner and edge solitons. Here $\mu=1$.
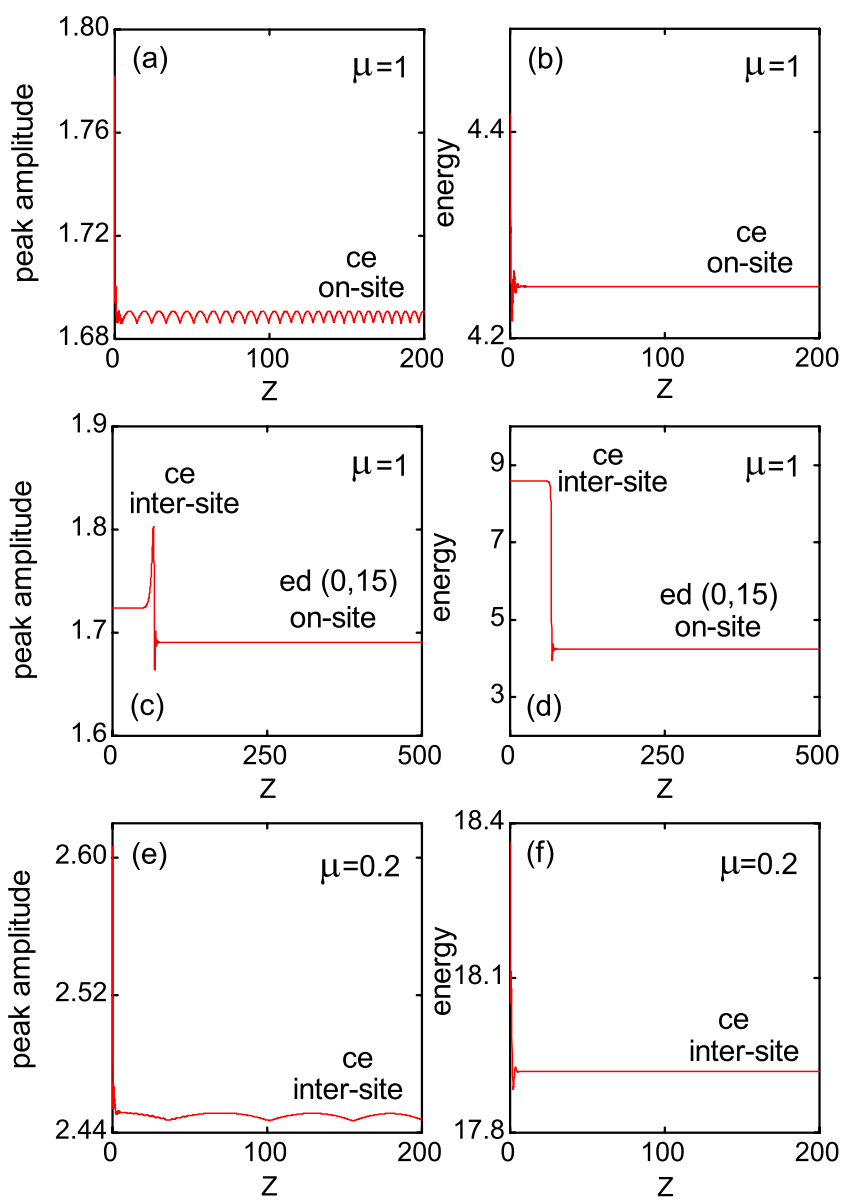

FIG. 10. (Color online) Evolution of (a),(c),(e) on-site and intersite peak amplitudes and (b),(d),(f) energies for stable (a),(b) onsite and (e),(f) intersite central modes, and for unstable (c),(d) intersite central modes. Here $\varepsilon=3.5$ and $\mu=1$ in (a)-(d), and $\varepsilon=1.5$ and $\mu=0.2$ in (e)-(f).

stationary solutions, whereas the intersite solitons, for which the linear stability analysis predicts the existence of a nonzero real part of the perturbation eigenvalue, are found to be indeed unstable in numerical propagation simulated without adding a white noise at the input. As typical examples, in Figs. 11 and 12 we show the evolution of the soliton peak amplitude and energy vs propagation distance for both stable on-site and unstable two-peaked intersite corner and edge solitons.

As the next step, we analyze systematically the possible outcomes of the evolution of the unstable double-peak intersite solitons localized at the corner or at the edge or the center, which are propagated without an input noise. We fix the parameter $\mu=1$ and vary the cubic gain parameter $\varepsilon$. Recall that for our choice of parameters the intersite corner and edge solitons exist in the intervals $\varepsilon_{\text {low }}=3.185<\varepsilon$ $<3.743=\varepsilon_{\text {upp }}$, and $\varepsilon_{\text {low }}=3.262<\varepsilon<3.746=\varepsilon_{\text {upp }}$, respectively. We find that, for relatively low values of the cubic gain parameter in the vicinity of the minimum permitted value $\varepsilon_{\text {low }}$, the input unstable intersite corner and edge solitons decay. If we increase the cubic gain, e.g., to the value $\varepsilon=3.3$, the relatively low-peak-amplitude unstable intersite solitons localized in the corner and at the edge eventually 

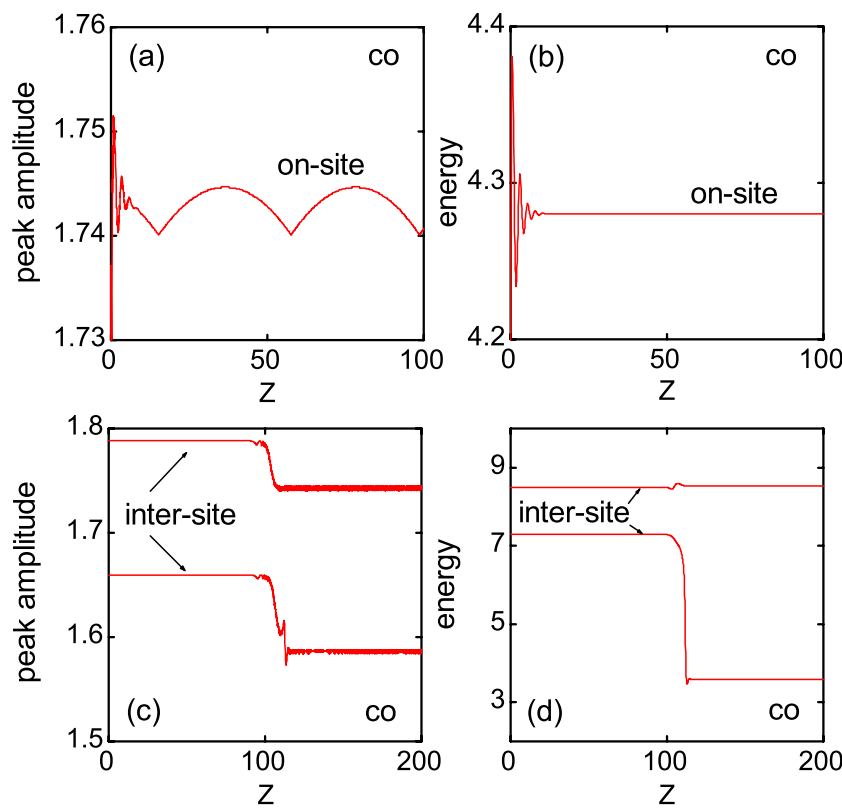

FIG. 11. (Color online) Evolution of the stable on-site and unstable intersite peak amplitudes (a),(c) and energies (b),(d) versus propagation distance for the corner solitons. Here $\mu=1, \varepsilon=3.5$ $[(\mathrm{a}),(\mathrm{b})$ and upper curves in (c),(d)], and $\varepsilon=3.3$ [lower curves in (c), (d)].

reshape to moving low-energy stable on-site $(0,0)$ corner and $(0,15)$ edge solitons, which correspond to the same set of parameters of the GL equation [see the lower curves in Figs. 11(c), 11(d), 12(c), 12(d), 13(a), and 13(c)]. However, for larger values of the cubic gain (e.g., for $\varepsilon=3.5$ ) the relatively high-peak-amplitude unstable intersite corner and edge solitons split into two stable on-site corner and edge solitons, moving in opposite directions and centered in two different lattice sites $(0,0)$ and $(1,1)[(0,15)$ and $(1,15)]$. These
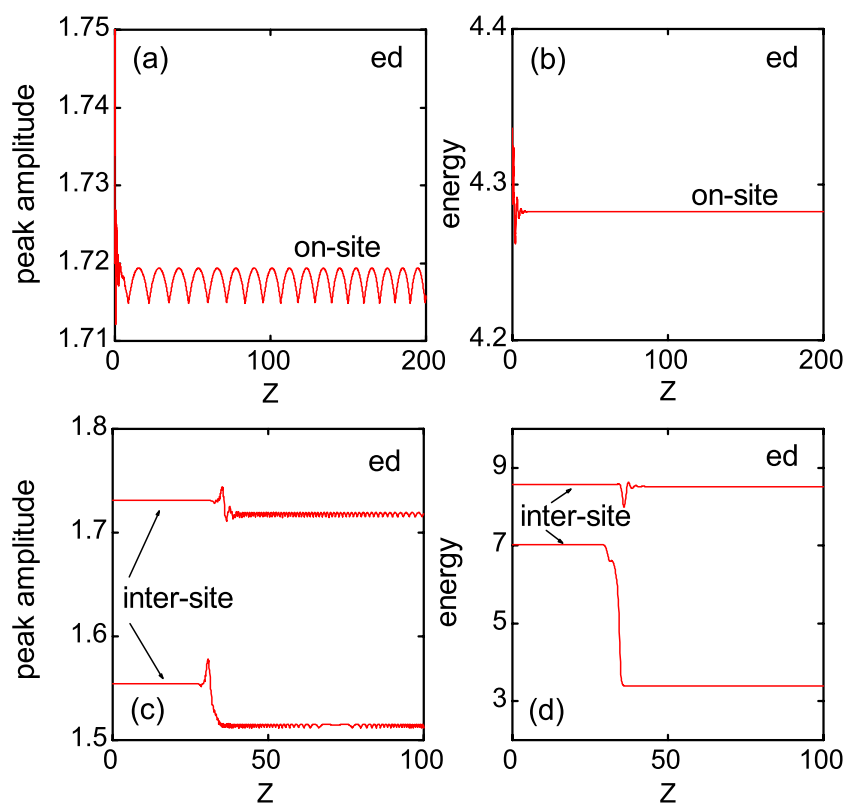

FIG. 12. (Color online) The same as in Fig. 11 but for the edge solitons.
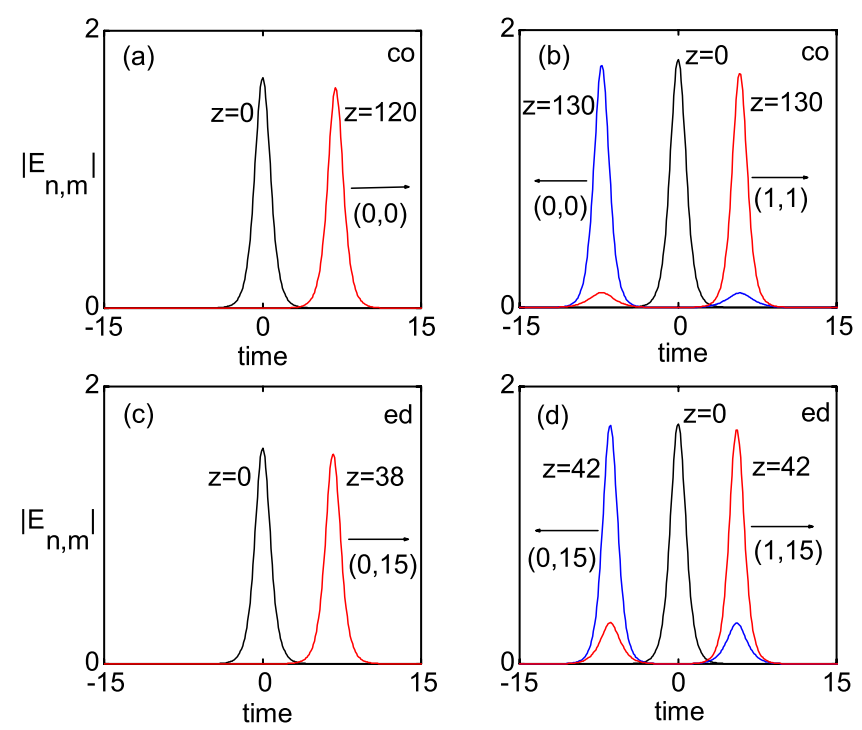

FIG. 13. (Color online) Reshaping of the relatively lowamplitude unstable intersite (a) $(0,0)$ corner and (c) $(0,15)$ edge solitons into moving low-energy on-site corner and edge solitons, respectively, centered at the same lattice sites, and splitting of the relatively high-amplitude unstable intersite (b) corner and (d) edge solitons into two on-site solitons moving in opposite directions and localized at different lattice sites. Here $\mu=1, \varepsilon=3.3$ (a), (c), and $\varepsilon=3.5$ (b), (d).

emerging pairs of stable on-site solitons [see the upper curves in Figs. 11(c), 11(d), 12(c), 12(d), 13(b), and 13(d)] correspond to the same set of parameters of the GL equation. Finally, for relatively large values of the cubic gain parameter in the vicinity of the maximum permitted value $\varepsilon_{\text {upp }}$, the input unstable intersite solitons generate expanding patterns composed of two fronts moving in opposite directions along the temporal axis (the corresponding energy increases indefinitely). Concerning the instability scenarios of the two-peak intersite solitons located deep inside the lattice (the bulk mode) we have found that the unstable intersite $(15,15)$ center soliton decays into a stable on-site $(0,15)$ edge soliton [see Figs. 10(c) and 10(d) for the evolution of the soliton peak amplitude and energy].

\section{CONCLUSIONS}

In the framework of the continuous-discrete cubic-quintic Ginzburg-Landau model, we have analyzed the existence and stability of spatiotemporal dissipative solitons in twodimensional photonic lattices with cubic nonlinear response in the presence of gain and loss, which appear due to optical amplifiers and saturable absorbers. We have demonstrated that the presence of gain and loss supports the existence of novel classes of stable continuous-discrete two-dimensional spatiotemporal Ginzburg-Landau solitons, which can also be termed dissipative discrete light bullets. These classes of nonlinear localized modes include both the bulk lattice solitons localized in the center of the two-dimensional lattice and the surface lattice solitons which are localized in the lattice corners or at the edges. We have studied the soliton 
linear stability and also described several unique instabilityinduced scenarios of the dissipative lattice solitons, such as the formation of a moving stable soliton and the occurrence of two solitons, moving in opposite directions, centered at two adjacent sites of the photonic lattice.

\section{ACKNOWLEDGMENTS}

This work was supported in part by the Deutsche Forschungsgemeinschaft (DFG, Bonn) and by the Australian Research Council.
[1] W. J. Tomlinson, Opt. Lett. 5, 323 (1980); V. M. Agranovich, V. S. Babichenko, and V. Y. Chernyak, JETP Lett. 32, 512 (1980).

[2] V. K. Fedyanin and D. Mihalache, Z. Phys. B: Condens. Matter 47, 167 (1982); D. Mihalache, R. G. Nazmitdinov, and V. K. Fedyanin, Phys. Scr. 29, 269 (1984).

[3] U. Langbein, F. Lederer, and H.-E. Ponath, Opt. Commun. 46, 167 (1983).

[4] G. I. Stegeman, C. T. Seaton, J. Chilwell, and S. D. Smith, Appl. Phys. Lett. 44, 830 (1984).

[5] N. N. Akhmediev, V. I. Korneyev, and Y. V. Kuzmenko, Zh. Eksp. Teor. Fiz. 88, 107 (1985) [Sov. Phys. JETP 61, 62 (1985)].

[6] F. Lederer and D. Mihalache, Solid State Commun. 59, 151 (1986); D. Mihalache, D. Mazilu, and F. Lederer, Opt. Commun. 59, 391 (1986); D. Mihalache et al., Opt. Lett. 12, 187 (1987).

[7] D. Mihalache, M. Bertolotti, and C. Sibilia, Prog. Opt. 27, 229 (1989).

[8] A. D. Boardman, P. Egan, F. Lederer, U. Langbein, and D. Mihalache, in Nonlinear Surface Electromagnetic Phenomena, edited by H.-E. Ponath and G. I. Stegeman (Elsevier Science, Amsterdam, 1991), pp. 73-287.

[9] F. Lederer, L. Leine, R. Muschall, T. Peschel, C. SchmidtHattenberger, U. Trutschel, A. D. Boardman, and C. Wächter, Opt. Commun. 99, 95 (1993).

[10] K. G. Makris, S. Suntsov, D. N. Christodoulides, G. I. Stegeman, and A. Haché, Opt. Lett. 30, 2466 (2005).

[11] S. Suntsov, K. G. Makris, D. N. Christodoulides, G. I. Stegeman, A. Haché, R. Morandotti, H. Yang, G. Salamo, and M. Sorel, Phys. Rev. Lett. 96, 063901 (2006).

[12] M. I. Molina, R. A. Vicencio, and Yu. S. Kivshar, Opt. Lett. 31, 1693 (2006).

[13] Yu. S. Kivshar and G. P. Agrawal, Optical Solitons: From Fibers to Photonic Crystals (Academic Press, San Diego, 2003).

[14] Y. V. Kartashov, V. A. Vysloukh, D. Mihalache, and L. Torner, Opt. Lett. 31, 2329 (2006); Y. V. Kartashov, L. Torner, and V. A. Vysloukh, ibid. 31, 2595 (2006).

[15] Y. V. Kartashov, F. Ye, V. A. Vysloukh, and L. Torner, Opt. Lett. 32, 2260 (2007).

[16] Y. V. Kartashov, V. A. Vysloukh, and L. Torner, Phys. Rev. A 76, 013831 (2007).

[17] M. I. Molina, Y. V. Kartashov, L. Torner, and Yu. S. Kivshar, Opt. Lett. 32, 2668 (2007).

[18] M. I. Molina, Y. V. Kartashov, L. Torner, and Yu. S. Kivshar, Phys. Rev. A 77, 053813 (2008).

[19] C. R. Rosberg, D. N. Neshev, W. Krolikowski, A. Mitchell, R. A. Vicencio, M. I. Molina, and Yu. S. Kivshar, Phys. Rev. Lett. 97, 083901 (2006).

[20] E. Smirnov, M. Stepić, C. E. Rütter, D. Kip, and V. Shandarov,
Opt. Lett. 31, 2338 (2006).

[21] B. Alfassi, C. Rotschild, O. Manela, M. Segev, and D. N. Christodoulides, Phys. Rev. Lett. 98, 213901 (2007).

[22] A. Szameit, Y. V. Kartashov, F. Dreisow, M. Heinrich, T. Pertsch, S. Nolte, A. Tünnermann, V. A. Vysloukh, and L. Torner, Opt. Lett. 33, 1132 (2008).

[23] X. Wang, A. Samodurov, and Z. Chen, Opt. Lett. 33, 1240 (2008).

[24] S. Suntsov et al., J. Nonlinear Opt. Phys. Mater. 16, 401 (2007); F. Lederer, G. I. Stegeman, D. N. Christodoulides, G. Assanto, M. Segev, and Y. Silberberg, Phys. Rep. 463, 1 (2008); Yu. S. Kivshar, Laser Phys. Lett. 5, 703 (2008).

[25] D. Mihalache, D. Mazilu, F. Lederer, and Yu. S. Kivshar, Phys. Rev. A 77, 043828 (2008).

[26] N. K. Efremidis and D. N. Christodoulides, Phys. Rev. E 67, 026606 (2003)

[27] E. A. Ultanir, G. I. Stegeman, D. Michaelis, C. H. Lange, and F. Lederer, Phys. Rev. Lett. 90, 253903 (2003).

[28] U. Peschel, O. Egorov, and F. Lederer, Opt. Lett. 29, 1909 (2004).

[29] N. K. Efremidis, D. N. Christodoulides, and K. Hizanidis, Phys. Rev. A 76, 043839 (2007).

[30] A. B. Aceves, C. De Angelis, A. M. Rubenchik, and S. K. Turitsyn, Opt. Lett. 19, 329 (1994); E. W. Laedke, K. H. Spatschek, and S. K. Turitsyn, Phys. Rev. Lett. 73, 1055 (1994); A. V. Buryak and N. N. Akhmediev, IEEE J. Quantum Electron. 31, 682 (1995).

[31] Z. Xu, Y. V. Kartashov, L. C. Crasovan, D. Mihalache, and L. Torner, Phys. Rev. E 70, 066618 (2004).

[32] Yu. V. Bludov and V. V. Konotop, Phys. Rev. E 76, 046604 (2007).

[33] N. C. Panoiu, B. A. Malomed, and R. M. Osgood, Phys. Rev. A 78, 013801 (2008); N. C. Panoiu, R. M. Osgood, and B. A. Malomed, Opt. Lett. 31, 1097 (2006).

[34] Q. E. Hoq, R. Carretero-Gonzales, P. G. Kevrekidis, B. A. Malomed, D. J. Frantzeskakis, Yu. V. Bludov, and V. V. Konotop, Phys. Rev. E 78, 036605 (2008).

[35] C. J. Benton, A. V. Gorbach, and D. V. Skryabin, Phys. Rev. A 78, 033818 (2008).

[36] H. Leblond, B. A. Malomed, and D. Mihalache, Phys. Rev. A 77, 063804 (2008).

[37] F. Kh. Abdullaev, Yu. V. Bludov, S. V. Dmitriev, P. G. Kevrekidis, and V. V. Konotop, Phys. Rev. E 77, 016604 (2008).

[38] D. Mihalache, D. Mazilu, F. Lederer, and Yu. S. Kivshar, Opt. Express 15, 589 (2007).

[39] D. Mihalache, D. Mazilu, F. Lederer, and Yu. S. Kivshar, Opt. Lett. 32, 2091 (2007).

[40] K. G. Makris, J. Hudock, D. N. Christodoulides, G. Stegeman, O. Manela, and M. Segev, Opt. Lett. 31, 2774 (2006). 
[41] R. A. Vicencio, S. Flach, M. I. Molina, and Yu. S. Kivshar, Phys. Lett. A 364, 274 (2007).

[42] H. Susanto, P. G. Kevrekidis, B. A. Malomed, R. CarreteroGonzález, and D. J. Frantzeskakis, Phys. Rev. E 75, 056605 (2007).

[43] X. Wang, A. Bezryadina, Z. Chen, K. G. Makris, D. N. Christodoulides, and G. I. Stegeman, Phys. Rev. Lett. 98, 123903 (2007).

[44] A. Szameit, Y. V. Kartashov, F. Dreisow, T. Pertsch, S. Nolte, A. Tünnermann, and L. Torner, Phys. Rev. Lett. 98, 173903 (2007).

[45] I. S. Aranson and L. Kramer, Rev. Mod. Phys. 74, 99 (2002).

[46] Dissipative Solitons, edited by N. Akhmediev and A. Ankiewicz, Lecture Notes in Physics Vol. 661 (Springer,
Berlin, 2005).

[47] N. N. Rosanov, Spatial Hysteresis and Optical Patterns (Springer, Berlin, 2002).

[48] J. M. Ortega and W. C. Rheinboldt, Iterative Solution of Nonlinear Equations in Several Variables (Academic Press, New York, 1970), p. 182.

[49] Y. Silberberg, Opt. Lett. 15, 1282 (1990).

[50] B. A. Malomed, D. Mihalache, F. Wise, and L. Torner, J. Opt. B: Quantum Semiclassical Opt. 7, R53 (2005).

[51] D. Mihalache, D. Mazilu, Yu. S. Kivshar, and F. Lederer, Opt. Express 15, 10718 (2007).

[52] D. Mihalache, D. Mazilu, F. Lederer, and Yu. S. Kivshar, Opt. Lett. 32, 3173 (2007). 